\title{
A Remote Online Microscopy Lab as a Tool for K-12 Science Education and a Platform for Teacher-Scientist Partnerships
}

\author{
E. Ferrin ${ }^{1}$, A. Pratt ${ }^{1}$, K. Jona ${ }^{1}$ \\ 1. Office of STEM Education Partnerships, School of Education and Social Policy, Northwestern \\ University, 2120 Campus Drive, Evanston, Illinois, 60208.
}

Founded in 2006, the Office of STEM Education Partnerships is focused on making cutting-edge STEM research and resources accessible and relevant to the K-12 classroom. Our staff consists of scientists, education researchers, curriculum development professionals, and experienced K-12 educators, merging STEM content knowledge with the pedagogical expertise needed to design today's STEM teaching and learning models. Our goal is to create effective, scalable, and sustainable STEM outreach and education programs that prepare students to be tomorrow's innovators and problem solvers.

The original mission of OSEP was to create a platform for Northwestern University faculty to build education and outreach programs based on their research. Today, OSEP not only lowers barriers to faculty engagement, it builds partnerships with industry, government, and communities to create new opportunities for K-12 education and outreach. Through these partnerships and supported by our staff expertise, OSEP has developed powerful and effective education programs that have been successfully adopted by many K-12 schools. OSEP is currently serving over 140 schools, 368 teachers, and approximately 30,000 students in the Chicago area.

One example of how OSEP has partnered with researchers to create effective K-12 science curricula is the remote online labs project (http://osep.northwestern.edu/projects/ilab). We have created remote online laboratories that enable students and educators to use real instruments, rather than simulations, to carry out experiments from anywhere at any time. Unlike conventional facilities, these labs can be shared and accessed widely by audiences both locally and globally that might not otherwise have the resources to purchase and operate costly or delicate lab equipment.

The Powder X-Ray Diffraction iLab is one of our newest modules. Students study the crystalline lattice structure of a silicon sample by remotely accessing an X-ray diffractometer located at Northwestern University. Following a series of steps students can investigate how to characterize the sample crystalline structure and determine the unit cell dimensions. The interface embeds scaffolding to help students practice good experimental design and data analysis skills. Careful attention is paid to aligning with national science and professional development standards.

Evaluation and efficacy studies of iLabs has shown that remote online labs can overcome many common school laboratory constraints, provide students with the opportunity to engage in more authentic scientific inquiry tasks, and support the development of epistemologies that are better aligned with those of authentic science. The design of our iLabs curriculum and interface emphasizes these authentic scientific inquiry modes. Data from our pilot test with high school students confirms that students using iLabs improve their scientific inquiry, reasoning and design skills. Further, our study of remote online labs and simulations showed that remote labs intrinsically provide support for students' authentic scientific inquiry and reasoning skill development. 
The success of OSEP makes a strong case that primary support for teacher-scientist partnerships be housed at the institutional level, rather than at the individual faculty level as has traditionally been done by funding agencies. In this session, we describe the OSEP model and provide iLabs as an example of a successful teacher-scientist partnership program, illustrating the value that OSEP delivers to Northwestern faculty, the university as a whole, and our many partner K-12 teachers, schools, and students.

Acknowledgements:

[1] We acknowledge our collaborators, the Northwestern University Materials Research Science and Engineering Center (MRSEC). This works is funded by the National Science Foundation under NSF Award Number DMR-1121262.

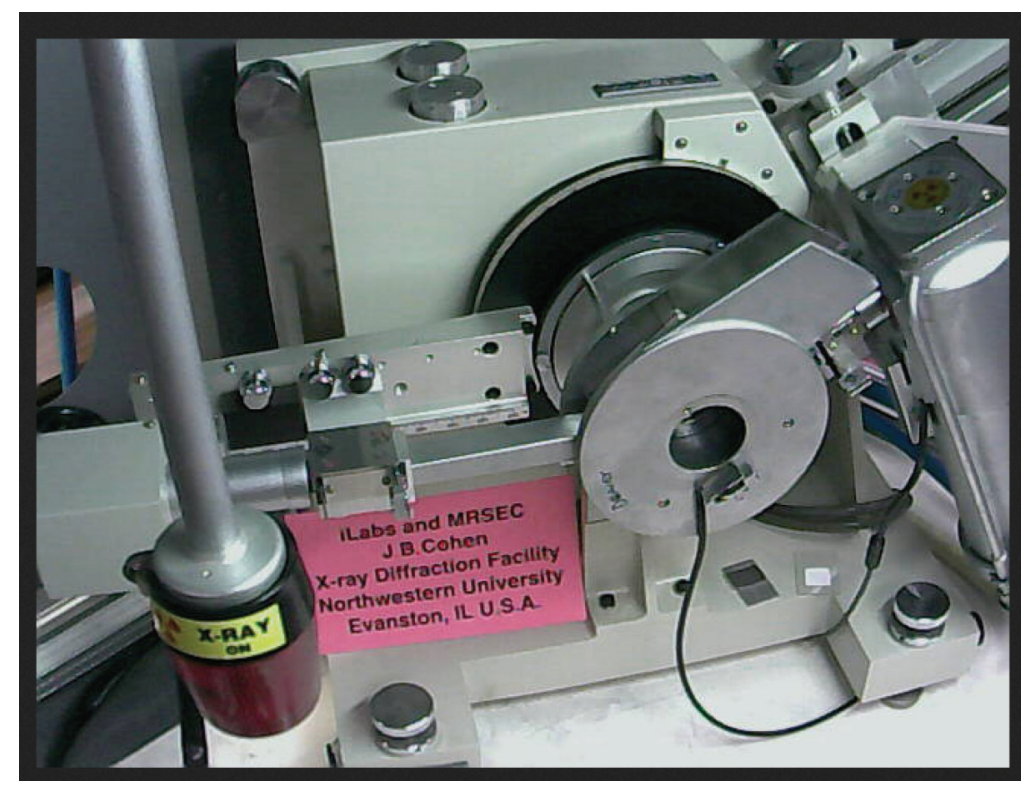

Figure 1: X-Ray diffractometer used in the Powder X-Ray Diffraction iLab.

Figure 2: User interface of the Powder X-Ray Diffraction iLab.
¡LabCentral ${ }_{\mid \text {Real Labs. Real Learning. }}$

Powder X-ray Diffraction iLab

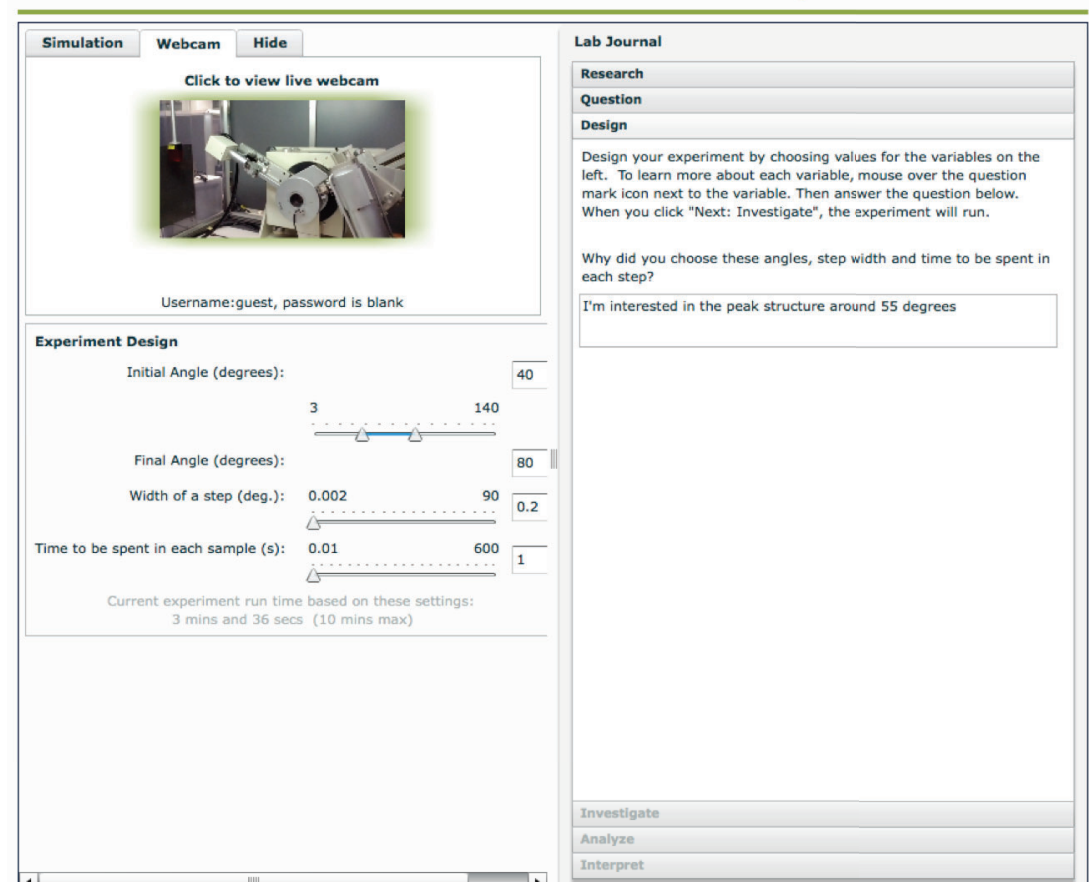

